\title{
Control theory in biology and medicine
}

\author{
Introduction to the special issue
}

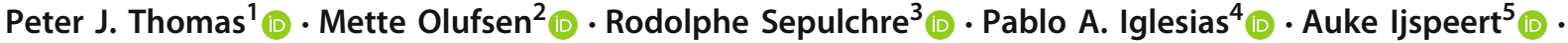 Manoj Srinivasan ${ }^{6}$ (I)}

Published online: 30 January 2019

(c) Springer-Verlag GmbH Germany, part of Springer Nature 2019

\begin{abstract}
From September-December 2017, the Mathematical Biosciences Institute at Ohio State University hosted a series of workshops on control theory in biology and medicine, including workshops on control and modulation of neuronal and motor systems, control of cellular and molecular systems, control of disease / personalized medicine across heterogeneous populations, and sensorimotor control of animals and robots. This special issue presents tutorials and research articles by several of the participants in the MBI workshops.
\end{abstract}

Control theory is a mathematically oriented discipline within engineering that concerns the design and analysis of

\footnotetext{
${ }^{1}$ Contrary to popular belief, James Watt did not invent the Watt governor; the mechanism was in use for wind and water mills well before he adapted it to pressure regulation in steam engines in the late $1700 \mathrm{~s}$ [37].
}

Peter J. Thomas

pjthomas@case.edu

https://case.edu/math/thomas/

Mette Olufsen

msolufse@ncsu.edu

https://olufsen.wordpress.ncsu.edu

Rodolphe Sepulchre

r.sepulchre@eng.cam.ac.uk

http://www-control.eng.cam.ac.uk/Main/RodolpheSepulchre

Pablo A. Iglesias

pi@jhu.edu

https://www.ece.jhu.edu/cscl/

Auke Ijspeert

auke.ijspeert@epfl.ch

https://biorob.epfl.ch/people/ijspeert

Manoj Srinivasan

srinivasan.88@osu.edu

http://movement.osu.edu

1 Department of Mathematics, Applied Mathematics and Statistics, Case Western Reserve University, Cleveland, Ohio, USA

2 Department of Mathematics, North Carolina State University, Raleigh, North Carolina, USA systems for the regulation of physical devices [35]. Classical applications in mechanical and aerospace engineering range from Watt's centrifugal governor to autopilots in modern commercial aircraft. ${ }^{1}$ Control theory has a broad scope, encompassing problems as diverse as system identification, state and parameter estimation, analysis of nonlinear feedback control systems, and optimal control.

The analogy between mechanical control and regulation in biological systems was articulated in Norbert Wiener's 1948 book Cybernetics: or Control and Communication in the Animal and the Machine [38]. This analogy has proven fruitful in many domains of physiology (including computational neuroscience). In a 1954 paper on a closed-loop control model of the respiratory chemostat, Grodins and colleagues put it thus: "The essence of physiology is regulation. It is this concern with purposeful system responses which distinguishes physiology from biophysics and biochemistry. Thus, physiologists study the regulation of breathing, of cardiac output, of blood pressure, of water balance, of body temperature and of a host of other biological phenomena. In recent years, stim-

\footnotetext{
3 Engineering Department, Cambridge University, Cambridge, UK

4 Department of Electrical and Computer Engineering, Johns Hopkins University, Baltimore, Maryland, USA

5 Institute of Bioengineering, EPFL, Lausanne, Switzerland

6 Department of Mechanical and Aerospace Engineering, Ohio State University, Columbus, Ohio, USA
} 
ulated particularly by the practical demands of World War II, a closely related branch of physical science has emerged. It is concerned with the theory, design, and operation of manmade regulators and servomechanisms. It is usually referred to as control system engineering, but it could very well be called the physiology of physical systems" [14].

The sentiment articulated by Wiener and Grodins was echoed in the founding mission statement of this journal (in 1961, under its original title Kybernetik): "The concepts of transmission of information, processing of information and automatic control engineering originated within technology and physics. Today, however, these concepts have also found application in the biological sciences." Application of control theoretic ideas to a variety of biological systems remains a central topic for Biological Cybernetics.

Many aspects of the central nervous system may fruitfully be studied from such a perspective. For instance, as the authors of [15] in this special issue write: The nervous system acts as a controller for locomotion. Indeed, central pattern generators are an important example of control systems, and many analyses of motor control systems have appeared in Biological Cybernetics $[13,16,18,33,36]$. As an example, the respiratory rhythm, produced by activity of neural circuits in the brainstem, has been studied as a control problem for many years $[4,11]$; apnea, or recurring prolonged interruption of the rhythm, has been considered as a controller instability $[23,25]$.

Control theory in biology and medicine remains an active area of development; for a recent monograph see [20]. From September-December 2017, the Mathematical Biosciences Institute (MBI) at Ohio State University hosted an emphasis semester on this theme, including workshops on "Control and Modulation of Neuronal and Motor Systems (I)," "Control of Cellular and Molecular Systems (II)," "Control of Disease: Personalized Medicine across Heterogeneous Populations (III)," and "Sensori-motor Control of Animals and Robots (IV)" (Fig. 1). Biological Cybernetics is pleased to present this special issue as a collection of papers by several of the participants in the MBI workshops.

The papers cover a broad range of topics within control theory. Optimization problems arising in the design of efficient controllers for neuronal, cardiac, and motor systems are discussed in $[2,7,28,29]$. Optimal feedback controls ${ }^{2}$ intrinsic to the organism are analyzed for homeostasis of blood pressure [39] and neuronal firing rates [27]. One paper [24] discusses optimizing a new approach to regulation of blood sugar in a diabetes model. Another broad theme concerns parameter identifiability and parameter estimation: both esti-

2 The importance of understanding feedback in biological systems was emphasized by workshop II participant H. El Samad (UCSF) who declared "This is the dawning of the age of feedback control in biology." mating physiological parameters from experimental data $[8,32]$ and exploring how an organism might estimate parameters related to the stability of its own movements [15]. Controllability of systems with nonlinear dynamics is discussed in the context of ultrasensitivity and robust rhythm generation [10], and robust generation of waves in excitable media [3]. The role of the nervous system itself as a controller is emphasized in $[15,19]$.

Tutorials The MBI workshop series began with a series of tutorials intended to help quantitative biologists and applied mathematicians new to control theory find their way into the field. Ken Loparo (Case Western Reserve University) gave an overview of linear systems theory; Rodolphe Sepulchre (University of Cambridge) gave a tutorial on feedback, sensitivity, and excitability; Robert Parker (University of Pittsburgh) introduced model predictive control; and Terence Sanger lectured on nonlinear filters. Videos of these tutorials and many of the research talks were archived. ${ }^{3}$

In a similar spirit, we begin this special issue with three tutorial reviews solicited from speakers at the workshops. The tutorials form a series addressing biological systems that are subject to an increasing number of control inputs: control through a single input such as an injected current [29]; a dual control system such as parallel control of synaptic gain and intrinsic excitability in a nerve cell [27]; and the control of waves in excitable media subject to three key parameters, time-scale separation, space-scale separation, and activation threshold [3].

The first tutorial, Phase reduction and phase-based optimal control for biological systems, by Monga et al. [29] surveys open-loop control methods based on reduction of high-dimensional dynamical systems models to lowerdimensional effective models. In many situations, highdimensional dynamical systems can be better understood when low-dimensional organizing structure can be identified within them. For instance, rhythmic phenomena such as the repetitive firing of nerve or cardiac cells may be described by systems of ODEs or PDEs with trajectories converging to a low-dimensional manifold containing a stable limit cycle. A limit cycle trajectory can be parametrized by a single-phase variable representing the progression around the periodic limit cycle orbit. This "asymptotic phase" variable can be extended to points within the basin of attraction of the limit cycle, giving a useful one-dimensional representation of the full dynamics. Isostables generalize the notion of isochrons to systems with a fixed point rather than a limit cycle [26,40], and provide dimension reduction (by adiabatic elimination of fast dynamics) for excitable systems. Control of periodic orbits based on standard phase reduction is limited to controls under which the controlled orbit remains within a small

\footnotetext{
${ }^{3}$ Seehttps : / /mbi.osu. edu/event/?id=1062\#resources.
} 


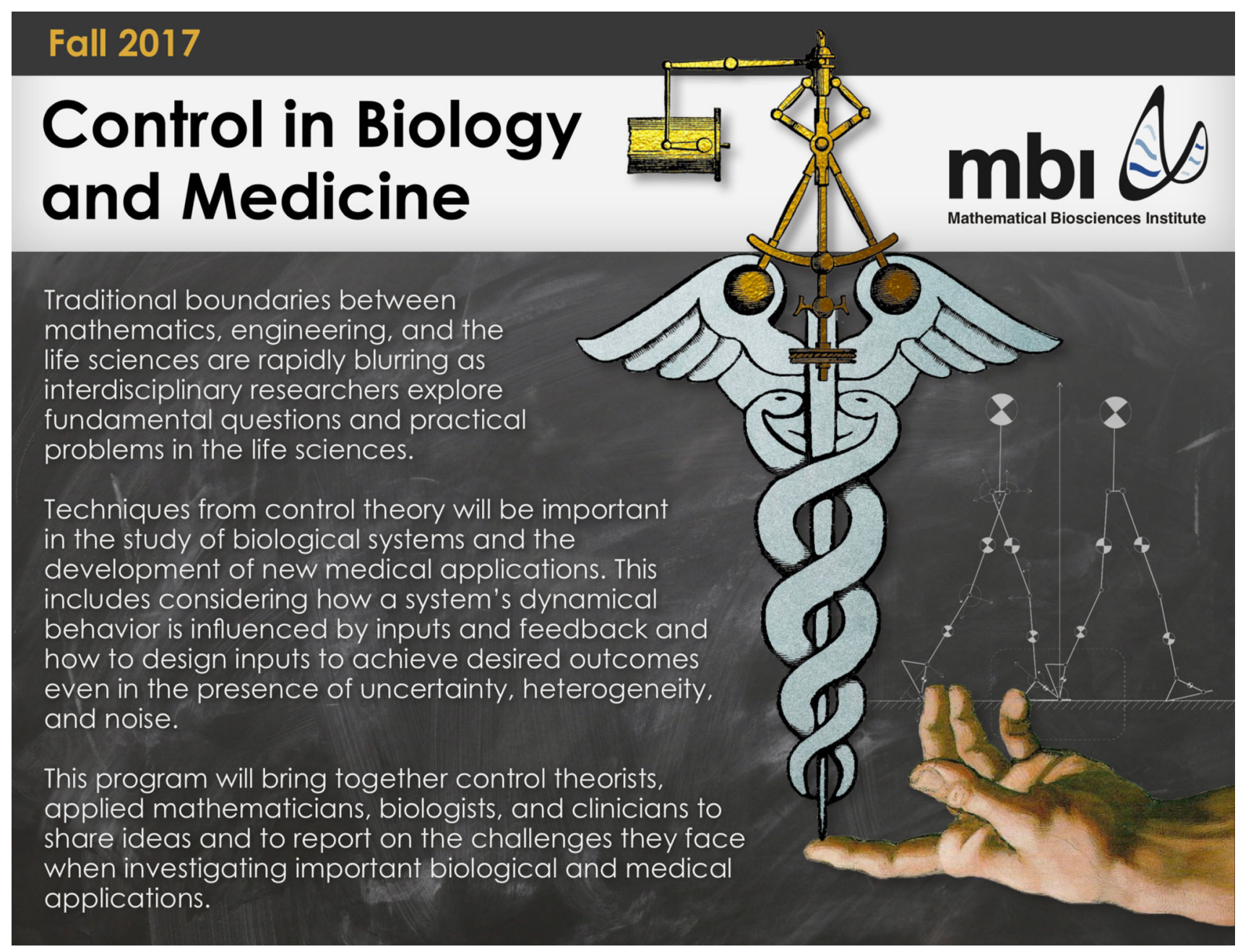

Fig. 1 Illustration from the Fall 2017 emphasis semester on Control Theory in Biology and Medicine at the Mathematical Biological Sciences Institute (MBI). Art credit: Will Gehring, Ohio State University/MBI. Used with permission

neighborhood of the limit cycle. The tutorial reviews standard phase reduction, isostables, and augmented phase reduction. As the authors illustrate, combining isostables and isochrons adds one or more coordinate directions transverse to the limit cycle, allowing the derivation of a broader range of control strategies. The authors demonstrate the superior effectiveness of these strategies, relative to those based on standard phase reduction, in cases where the periodic orbit has a nontrivial Floquet multiplier with magnitude close to unity. In this situation, there is at least one direction along which return to the limit cycle is slow enough that modest control inputs can significantly change the shape of the trajectory. Including the principal isostable along with the isochronal coordinate allows more precise control of timing by avoiding controls that push the trajectory far from the underlying periodic orbit.

The second tutorial by Miller and Cannon [27], concerns Combined mechanisms of neural firing rate homeostasis. Homeostasis is a fundamental control problem for biological systems: maintaining some quantity, such as body tempera- ture or blood glucose concentration, within prescribed limits. For these examples, minimizing the deviation from a set point is an appropriate goal. In contrast, neurons are subject to multiple forms of homeostasis. Firing rate adaptation should maintain the long-term average activity level of a nerve cell, but not so strictly as to prevent the short-term variations in firing frequency that are believed to encode (some of the) information in the nervous system. The tutorial [27] reviews recent work on dual control through mechanisms combining synaptic gain and intrinsic cellular excitability $[5,6]$, building on a framework developed by O'Leary and colleagues [30,31]. When two control mechanisms act on the same system, there is the potential for "wind-up" instability (imagine two heating/cooling systems regulated by linear thermostats, with equal gain but different set points, both trying to control the temperature of the same room). The tutorial reviews conditions under which neural homeostasis mechanisms can avoid wind-up instabilities, and jointly navigate gain and excitability under closed-loop control to find 
parameter values that maximize mutual information between neuronal inputs and outputs. Because the mutual information is sensitive to both the mean and variance of the firing rate, control through two mechanisms in concert proves to be superior to control through a single feedback mechanism alone. The authors also show how a dual control mechanism facilitates robust maintenance of a neural integrator (e.g., for maintaining eye position without visual feedback), and discuss a specific implementation of single and dual feedback control in terms of known molecular components of calcium ion signaling pathways in neurons.

Excitability, a form of ultrasensitivity, is a fundamental notion in biological cybernetics. Indeed Wiener [38, p. 17], proposed the study of "irritability" in cardiac muscle as a tractable and worthwhile alternative to irritability in the nervous system, and pursued this idea with his longtime collaborator Rosenblueth [34]. Excitable media play an important role in many physiological processes, for example, propagation of action potentials along the axon of a nerve cell [17] or propagation of a wave of activity along a chain of oscillators [21] (a traveling wave), generation of stable patterns of activation in a developing embryo [12] or in a neural network [1,9] (standing waves), and instability of cardiac activation patterns leading to atrial fibrillation [22] (spiraling waves). The final tutorial [3], Controlling excitable wave behaviors through the tuning of three parameters, by Bhattacharya and Iglesias, surveys how initiation of waves in excitable media can be controlled through three physical parameters: time-scale separation, space-scale separation, and activation threshold. The authors cover traveling, rotating, and standing waves, as well as wave reflection due to inhomogeneities in the wave medium or encounter with domain boundaries. The authors showcase a simple two-dimensional activator-inhibitor model, related to the FitzHugh-Nagumo equations, to illustrate the transitions between the different types of wave behavior under variation of the three different control parameters.

Articles The special issue continues with nine original articles written by participants. The first three investigate physiological control mechanisms, in rhythm generation by central circuits; in neuromotor control of a single limb; and in control of bipedal running or walking.

In Cellular switches orchestrate rhythmic circuits, Drion et al. [10] investigate the neural mechanisms underlying the robust generation of rhythms for various motor functions. They highlight an elegant possible mechanism of cellular switching based on a slow negative conductance that allows modulation of the individual participation of cells in the circuit rhythms and that can be externally controlled by neuromodulators. The cellular switch makes the circuits reconfigurable, robust, adaptable, and externally controllable. The authors illustrate these interesting properties with the generation of different rhythms in a circuit model of the crab stomatogastric ganglion.

Given properties of a network of neurons, we know how to approximate the dynamics of the network. One open problem in neuroscience is a predictive principle for why neural networks are organized the way they are in humans and other animals. To make progress on this question and in marked contrast to the current fashion of "deep learning" and large black-box multilayer neural networks trained on large complex data, the article $A$ normative approach to neuromotor control, by Berniker and Penny [2], describes a neural network at the other extreme: small (40 neurons), shallow (only three layers), and mostly understandable. Specifically, they consider a simple controller for a reaching task: The controller takes the current state of the "hand" as input and outputs muscle forces that move the hand toward its goal. They then approximate this linear controller by their small probabilistic neural network so that the output of the network produces the desired muscle force with high probability. Once their neural network is trained to approximate the controller, they find, remarkably, that it exhibits various phenomena previously observed in experiments, such as cosine tuning, population vectors correlated with reaching directions, and apparent oscillatory neural activity in point-to-point reaches. Consequently, they argue that these previous experimental observations are simply epiphenomena, emerging naturally from a regularized approximation of a linear controller in a single simple model. This line of research is promising: One could potentially apply similar techniques to derive biophysically grounded neural networks to control various other tasks, perhaps for much simpler organisms with well-characterized neural networks, and thereby compare the derived networks even more directly with what nature has produced.

Estimation problems form an important part of control theory, both parameter estimation and system identification, and state estimation for closed-loop control. In Locomotion: exploiting noise for state estimation, Guckenheimer and Javeed [15] investigate the hypothesis that in locomotory systems, such as bipedal walking, individuals actively perturb their own rhythmic motions in order to estimate stability parameters, such as Floquet exponents, which cannot be detected by observing noiseless periodic trajectories. In order for an organism to estimate the rate at which its own unperturbed locomotory rhythm would return to a nominal periodic orbit, the trajectory must first be displaced away from that orbit in a controlled fashion. The paper explores the time-versus-accuracy tradeoffs in time and accuracy for estimating Floquet multipliers using different types of stochastic processes as the source of perturbations. The authors suggest that heavy-tailed perturbation distributions, rather than Gaussian perturbations, are more effective for this purpose. They suggest experimental tests of this possibility, and for explor- 
ing the mechanisms by which organisms learn to stabilize rhythmic motions through practice.

We turn next to a series of articles addressing different aspects of system identification and parameter estimation for a critical physiological control system: the regulation of blood pressure. In Cardiovascular regulation in response to multiple hemorrhages: analysis and parameter estimation, Ciocanel et al. [8] study the cardiovascular response to a succession of blood withdrawals, analyzing the identifiability of different parameters in a quantitative physiologically based model of the cardiac regulatory system. They find that the identifiable parameters include those governing timing of cardiac contraction, systemic vascular resistance, and cardiac contractility. Of these, vascular resistance and cardiac contractility varied systematically over time.

One of the more significant bottlenecks in mathematical models of medicine and biology is the paucity of measured parameters. This will be of increasing importance as we move toward an era of personalized medicine, where patient-specific models are used as a basis for therapy. In Parameter subset selection techniques for problems in mathematical biology, Olsen et al. [32] consider the problem of parameter identifiability and parameter subset selection. Through a series of examples involving arterial blood pressure, they compare various methods and contrast their efficiency depending on the accuracy of initial parameter estimates.

Estimation problems typically involve optimization: One seeks the best estimate under some appropriate metric. Optimization also takes many other forms within control theory for biological systems, as illustrated by the remaining articles.

In Optimizing SGLT inhibitor treatment for diabetes with chronic kidney diseases, Layton [24] uses a detailed computational model of the kidney to study the effects of a novel diabetes therapy-sodium glucose transport (SGLT2) inhibitors-on kidney function. The SGLT2 is part of the body's mechanism for regulating blood sugar levels. This transporter normally lowers blood sugar levels by increasing the rate of secretion of urine in the kidneys, and may provide an alternative to insulin-based therapy for managing blood sugar levels. The study makes predictions about the effects of SGLT2 inhibition therapy on kidney function under normal physiology and in the presence of chronic kidney disease.

A much-vaunted advantage of the use of models in medical applications is that it enables experimentation without many of the hurdles, in terms of feasibility, costs, and ethical considerations, that would arise from in vivo experiments. In An optimal control approach for blood pressure regulation during head-up tilt, Williams et al. [39] use optimal control techniques to investigate the effect on the systemic blood flow, volume, and pressure changes in response to head-up tilt, a common test used to assess a patients ability to regulate blood pressure.

Optimal phase control of biological oscillators using augmented phase reduction, by Monga and Moehlis [28], details control algorithms for the optimal control of nonlinear oscillators on the basis of the augmented phase reduction theory (see also the tutorial review [29]). The method allows changing the phase using a minimum energy input and, as a novelty, the distance to the oscillator along a transverse direction. They apply their method to several classical oscillator models in the literature: the Andronov-Hopf bifurcation normal form, cardiac pacemaker cells, thalamic neurons, and circadian oscillators. The examples illustrate the applicability of the method in real problems such as treating jet lag and potentially reducing motors symptoms of Parkinsonian tremor.

In Spiking networks as efficient distributed controllers, Huang and Ching [19] address the question of tracking a reference trajectory of a linear state-space model with a spiking controller. The spike train is computed by minimizing a quadratic cost of the tracking error regularized by a penalty on the firing rate. The authors show that the resulting eventbased controller can be interpreted as the output of a recurrent neural network that combines rate coding and spike coding. The properties of the proposed controller are discussed from the general perspective of predictive neural coding.

Prospects We conclude the special issue with a Prospects piece by Chang and Paydarfar [7], Optimizing stimulus waveforms for electroceuticals, that reviews a variety of approaches to optimization of electrical stimulation waveforms for therapeutic purposes. The review emphasizes the importance of patient-to-patient variability and the need for optimization methods to be built into "electroceutical devices" directly. While traditional stimulus optimization is often limited to a small number of parameters, such as mean current, frequency, duration, and amplitude, advances in digital technology allow clinicians in principle to apply waveforms of unlimited complexity. In this context, the authors offer a broad survey of methods for seeking optimal control of therapeutic electrical stimulation waveforms, with examples drawn from deep brain stimulation for Parkinson's disease; defibrillation treatment of cardiac arrest; and cardioversion, or remediation of cardiac arrhythmia through reentrainment.

\section{References}

1. Amari S (1977) Dynamics of pattern formation in lateral-inhibition type neural fields. Biol Cybern 27(2):77-87

2. Berniker M, Penny S (2018) A normative approach to neuromotor control. Biol Cybern 1-10. https://doi.org/10.1007/00422-0180777-7 
3. Bhattacharya S, Iglesias PA (2018) Controlling excitable wave behaviors through the tuning of three parameters. Biol Cybern 110. https://doi.org/10.1007/s00422-018-0771-0

4. Botros S, Brace E (1990) Neural network implementation of a three-phase model of respiratory rhythm generation. Biol Cybern 63(2):143-153

5. Cannon J, Miller P (2016) Synaptic and intrinsic homeostasis cooperate to optimize single neuron response properties and tune integrator circuits. J Neurophysiol 116(5):2004-2022

6. Cannon J, Miller P (2017) Stable control of firing rate mean and variance by dual homeostatic mechanisms. J Math Neurosci 7(1):1

7. Chang J, Paydarfar D (2018) Optimizing stimulus waveforms for electroceuticals. Biol Cybern 1-9. https://doi.org/10.1007/s00422018-0774-x

8. Ciocanel MV, Docken SS, Gasper RE, Dean C, Carlson BE, Olufsen MS (2018) Cardiovascular regulation in response to multiple hemorrhages: analysis and parameter estimation. Biol Cybern 1-16. https://doi.org/10.1007/s00422-018-0781-y

9. Coombes S (2005) Waves, bumps, and patterns in neural field theories. Biol Cybern 93(2):91-108

10. Drion G, Franci A, Sepulchre R (2018) Cellular switches orchestrate rhythmic circuits. Biol Cybern 1-12. https://doi.org/10.1007/ s00422-018-0778-6

11. Feldman J, Cowan J (1975) Large-scale activity in neural nets II: a model for the brainstem respiratory oscillator. Biol Cybern 17(1):39-51

12. Gierer A, Meinhardt H (1972) A theory of biological pattern formation. Kybernetik 12(1):30-39

13. Gomi H, Kawato M (1992) Adaptive feedback control models of the vestibulocerebellum and spinocerebellum. Biol Cybern 68(2):105-114

14. Grodins FS, Gray JS, Schroeder KR, Norins AL, Jones RW (1954) Respiratory responses to $\mathrm{CO}_{2}$ inhalation. A theoretical study of a nonlinear biological regulator. J Appl Physiol 7(3):283-308

15. Guckenheimer J, Javeed A (2018) Locomotion: exploiting noise for state estimation. Biol Cybern 1-12. https://doi.org/10.1007/ s00422-018-0772-z

16. Haken H, Kelso JS, Bunz H (1985) A theoretical model of phase transitions in human hand movements. Biol Cybern 51(5):347-356

17. Hodgkin AL, Huxley AF (1952) A quantitative description of membrane current and its application to conduction and excitation in nerve. J Physiol 117(4):500-544

18. Hogan N (1985) The mechanics of multi-joint posture and movement control. Biol Cybern 52(5):315-331

19. Huang F, Ching S (2018) Spiking networks as efficient distributed controllers. Biol Cybern 1-12. https://doi.org/10.1007/s00422018-0769-7

20. Iglesias PA, Ingalls BP (2010) Control theory and systems biology. MIT Press, Cambridge

21. Ijspeert AJ (2001) A connectionist central pattern generator for the aquatic and terrestrial gaits of a simulated salamander. Biol Cybern 84(5):331-348

22. Keener JP (1986) A geometrical theory for spiral waves in excitable media. SIAM J Appl Math 46(6):1039-1056

23. Khoo MC (2000) Determinants of ventilatory instability and variability. Respir Physiol 122(2-3):167-182
24. Layton AT (2018) Optimizing SGLT inhibitor treatment for diabetes with chronic kidney diseases. Biol Cybern 1-10. https://doi. org/10.1007/s00422-018-0765-y

25. Longobardo G, Gothe B, Goldman M, Cherniack N (1982) Sleep apnea considered as a control system instability. Respir Physiol 50(3):311-333

26. Mauroy A, Mezić I, Moehlis J (2013) Isostables, isochrons, and Koopman spectrum for the action-angle representation of stable fixed point dynamics. Phys D Nonlinear Phenom 261:19-30

27. Miller P, Cannon J (2018) Combined mechanisms of neural firing rate homeostasis. Biol Cybern 1-13. https://doi.org/10.1007/ s00422-018-0768-8

28. Monga B, Moehlis J (2018) Optimal phase control of biological oscillators using augmented phase reduction. Biol Cybern 1-18. https://doi.org/10.1007/s00422-018-0764-z

29. Monga B, Wilson D, Matchen T, Moehlis J (2018) Phase reduction and phase-based optimal control for biological systems: a tutorial. Biol Cybern 1-36. https://doi.org/10.1007/s00422-018-0780-z

30. O'Leary T, Williams AH, Caplan JS, Marder E (2013) Correlations in ion channel expression emerge from homeostatic tuning rules. Proc Natl Acad Sci 110(28):E2645-E2654

31. O'Leary T, Williams AH, Franci A, Marder E (2014) Cell types, network homeostasis, and pathological compensation from a biologically plausible ion channel expression model. Neuron 82(4):809-821

32. Olsen CH, Ottesen JT, Smith RC, Olufsen MS (2018) Parameter subset selection techniques for problems in mathematical biology. Biol Cybern 1-18. https://doi.org/10.1007/s00422-018-0784-8

33. Prablanc C, Echallier J, Komilis E, Jeannerod M (1979) Optimal response of eye and hand motor systems in pointing at a visual target. Biol Cybern 35(2):113-124

34. Rosenblueth A, Wiener N (1946) The mathematical formulation of the problem of conduction of impulses in a network of connected excitable elements specifically in cardiac muscle. Arch Del Instit De Cardiologia De Mexico 16:205-265

35. Sontag ED (2013) Mathematical control theory: deterministic finite dimensional systems, vol 6. Springer, Berlin

36. Uno Y, Kawato M, Suzuki R (1989) Formation and control of optimal trajectory in human multijoint arm movement. Biol Cybern 61(2):89-101

37. Von Tunzelmann GN (1978) Steam power and British industrialization to 1860. Oxford University Press, Oxford

38. Wiener N (1948) Cybernetics: or control and communication in the animal and the machine. MIT Press, Cambridge

39. Williams ND, Mehlsen J, Tran HT, Olufsen MS (2018) An optimal control approach for blood pressure regulation during head-up tilt. Biol Cybern 1-11. https://doi.org/10.1007/s00422-018-0783-9

40. Wilson D, Moehlis J (2015) Extending phase reduction to excitable media: theory and applications. SIAM Rev 57(2):201-222

Publisher's Note Springer Nature remains neutral with regard to jurisdictional claims in published maps and institutional affiliations. 